\title{
Score Based Deadline Constrained Workflow Scheduling Algorithm for Cloud Systems
}

\author{
Ranjit Singh and Sarbjeet Singh \\ Computer Science and Engineering, Panjab University, Chandigarh, India
}

\begin{abstract}
Cloud Computing is the latest and emerging trend in information technology domain. It offers utility-based IT services to user over the Internet. Workflow scheduling is one of the major problems in cloud systems. A good scheduling algorithm must minimize the execution time and cost of workflow application along with QoS requirements of the user. In this paper we consider deadline as the major constraint and propose a score based deadline constrained workflow scheduling algorithm that executes workflow within manageable cost while meeting user defined deadline constraint. The algorithm uses the concept of score which represents the capabilities of hardware resources. This score value is used while allocating resources to various tasks of workflow application. The algorithm allocates those resources to workflow application which are reliable and reduce the execution cost and complete the workflow application within user specified deadline. The experimental results show that score based algorithm exhibits less execution time and also reduces the failure rate of workflow application within manageable cost. All the simulations have been done using CloudSim toolkit.
\end{abstract}

\section{KEYWORDS}

Cloud Computing, Score, QoS, Deadline Constraint

\section{INTRODUCTION}

Cloud computing [1] is the new and emerging trend in distributing computing that facilitate software applications, platform, and hardware infrastructures as a service. Cloud service providers offer these services based on customized Service Level Agreements (SLAs) which define user's required Quality of Service (QoS) parameters. Cloud computing reduces investment on various resources like hardware, software [2] and allow resources to be leased and released. It reduces initial investment, maintenance cost and operating cost. Cloud services are hosted on service provider's own infrastructure or on third party cloud infrastructure providers [3]. Mainly, three kinds of services are delivered: Platform as a Service (PaaS), Infrastructure as a Service (IaaS) and Software as a Service (SaaS). Cloud users use these services whenever needed according to their demand using pay-per-use model.

Besides many applications, cloud computing environment can be used for workflow execution also. Execution of a workflow involves workflow scheduling. Workflow scheduling involves mapping of workflow tasks with available resources in such a way that some predefined criteria is met. Workflow scheduling is well known NP-complete problem [4] and key issue in workflow management system. Moving workflows to Cloud computing enable us to exploit the benefits of cloud for workflow execution. Scheduling can be multi objective also. The multi objective nature of scheduling is more difficult to solve. Many heuristic and meta-heuristic approaches have been proposed by different researchers for workflow scheduling.

DOI : $10.5121 /$ ijccsa.2013.3603 
At present, workflow scheduling algorithms for cloud systems focus on two major parameters viz. cost and time. As cloud uses pay-as-you-go model, all services incur cost. Cost mainly dependents on QoS (Quality of Service) offered. Service providers charge higher for higher QoS and lower for lower QoS. Early and reliable execution of jobs is another important factor from cloud user's point of view, but it incurs more cost. Users may require earlier reliable completion of their workflow tasks within manageable cost along with other QoS requirements. These kinds of requirements make workflow scheduling on clouds more important and complex.

In this paper, a score based deadline constraint workflow scheduling algorithm has been proposed. The objective of the proposed scheduling algorithm is to develop workflow schedules which can execute the workflow within manageable cost and still satisfy the time constrained imposed by the user. The concept of score is inspired from Microsoft Windows, where it is used to define the computer hardware capability and performance requirement of software running on Windows operating system. Similar approach has been used here in the proposed algorithm. Score has been used to define the capability of virtual machines and minimum threshold performance requirements of workflow tasks.

The remainder of the paper is organized as follows: Section 2 describes workflows in Cloud computing. Section 3 presents related work. Section 4 describes motivation. Section 5 discusses the proposed algorithm. Experimental results are presented in Section 6. Finally, paper is concluded in Section 7.

\section{WORKFLOWS IN CLOUD}

Besides Cloud, workflows can be executed in Grids also. But due to the complexity of the environment in Grids, executing workflows in clouds is more promising as clouds offer less complex environment than grids. Cloud services like storage, compute and bandwidth are available at much lower costs. Scalability is the prime benefit which is achieved if workflows are moved to cloud. Scalability allow real-time provisioning of resources to meet workflow requirements. This makes it easy to satisfy Quality of Service (QoS) requirements of applications as opposite to grid approach which require prior reservation of resources in global multi-user environment. Workflow applications typically require complex execution environments which are difficult to implement on grids. Moreover, each grid site may have a different kind of configuration which results in an extra effort each time when an application is ported to a new site. Virtual machines allow developers to create fully customized execution environments, configured specifically for the application in hand.

Workflows are represented by a Directed Acyclic Graph (DAG) in which each node represents a task and the edge between corresponding nodes represents data dependency between tasks. Workflow scheduling is a key concern in workflow management systems. Workflow scheduling is the problem of mapping of workflow tasks on suitable resources while satisfying the constraints imposed by the user. Proper workflow scheduling can have significant impact on the performance of the workflow application [5]. Typical examples of workflow include bank account verification, insurance claim processing, e-business scenarios and online banking. Here we describe the example of bank account verification workflow processing to show the characteristics of workflows. In bank account verification, first of all, user enters his/her password, then system retrieve original password associated with account. User password is verified with original password. If password match with original password, system allow account access otherwise shows the warning message and request is denied. Figure 1 shows the simplified bank account verification process which can be modeled as a simple workflow with several steps. 


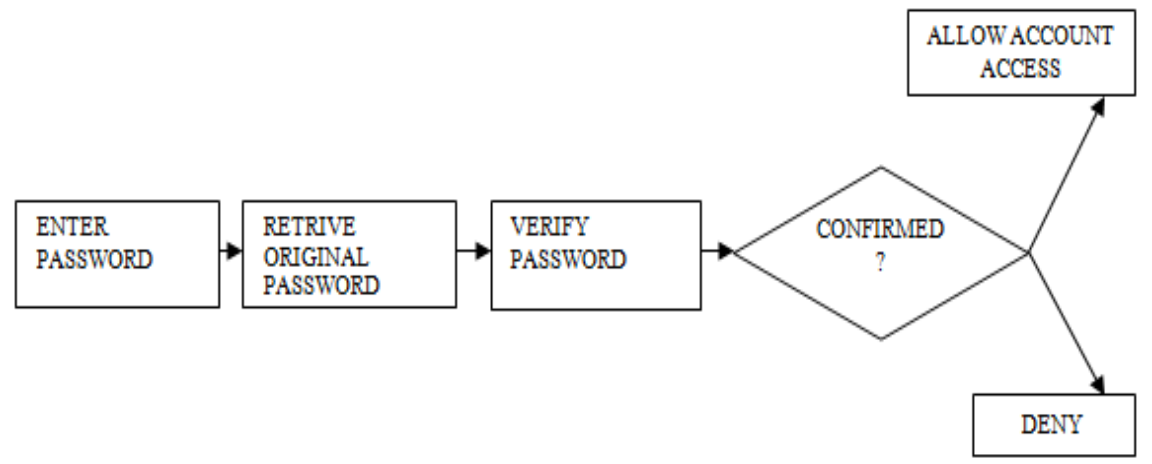

Figure 1. Simplified account verification workflow process

\section{RELATED WORK}

In 2006, Jia Yu et al. [6] proposed genetic based workflow scheduling algorithm which consider two QoS constraints: budget and deadline. Proposed genetic algorithm schedules workflow application either to minimize the makespan (while meeting user imposed deadline) or to minimize the execution cost (while meeting user imposed budget constraints). In this paper, authors mainly considered reservation based heterogeneous environment to handle deadline and budget. They compared the proposed genetic approach with existing genetic algorithms and their experimental results show that proposed technique not only reduces the execution time within specified deadline but also reduces execution cost within specified budget. This approach is directly applied to large heterogeneous environments on both balanced and unbalanced data structure for which most of the genetic algorithms cannot work.

In [7], scheduling strategy for multiple workflows, which consider multiple QoS constraints like budget and time, has been discussed. This strategy schedules multiple workflows with multiple QoS constraints. For algorithm performance, authors compared Multiple QoS Constrained Scheduling Strategy of Multi-Workflows (MWMQ) with dynamic scheduling algorithm RANK_HYBD. Results show that MWMQ has a higher success rate than RANK_HYBD because MQMW takes into account the QoS requirement while RANK_HYBD only considers the execution time.

[8] proposes workflow scheduling algorithm based on the heuristics of ant colony optimization (ACO) for scheduling large workflows along with various QoS parameters. Ant colony optimization (ACO) allow users to define their QoS preferences and specify their minimum QoS thresholds for workflow application. The main focus of this algorithm is to satisfy all user defined QoS constraints and improve the user specified QoS parameters.

[9] proposes a workflow scheduling algorithm which provides fault tolerance. Fault tolerance is achieved by using resubmission and replication of tasks. The main aim of proposed work is to provide a workflow schedule which satisfies deadline constraint in-spite of many failures which occur during workflow execution. Authors considered different number of workflows with different deadlines along with various types of faults. Results show that fault tolerant workflow scheduling algorithm performs better than the scheduling algorithms which do not consider any fault tolerance. The fault tolerant workflow scheduling (FTWS) shows better success rate when replication of tasks is considered as compared to cases where replication of tasks is not considered. 
In [10], authors propose a workflow scheduling algorithm based on heuristic of Particle Swarm Optimization (PSO) to provide schedule for workflow applications. PSO technique not only optimizes execution cost but also balances the load on the resources by distributing tasks over the resources. The proposed technique can be used for any number of resources and workflow tasks by simply increasing the dimensions of particles. Its performance has been analyzed by varying both the computation and communication cost and by comparing it with existing Best Resource Selection (BRS) algorithm. Analysis shows that PSO performs much better than the BRS in terms of cost and balances distribution of workloads onto resources.

In [11], authors proposed a compromised time-cost scheduling algorithm which considers instance intensive workflows. The CTC (compromised-time-cost) scheduling algorithm consider various aspects like background load, dynamic adjustment, rescheduling and checking of the tasks in order to deal with failure and uncompleted tasks. This algorithm is implemented over SwinDeW-C platform and its performance is compared with the deadline-MDP algorithm. Execution cost and makespan are the criteria for comparison. Simulation results show that CTC (compromised-time-cost) scheduling algorithm achieves lower cost than others while meeting the user defined deadlines.

In [12] a workflow scheduling algorithm for Hybrid Clouds is proposed which optimizes the cost of resources within deadline constraint. This algorithm select resources which should be rented from the public Cloud and aggregated to the private Cloud to provide enough processing power to execute a workflow within a given execution time. Designed algorithms can optimize cost while achieving specified deadline constraint. The performance of the algorithm has been compared with Greedy approach. Simulation results show that proposed technique not only reduces the execution time but also reduces the cost of resources which are rented from the private Cloud. In this, user can control the cost by simply adjusting the desired workflow execution time. Its cost knowledge and multi-core awareness allow users to reduce the execution time of workflow application as low as possible. This strategy also makes adjustments in the schedule when desired execution time is very low and provides fewer violations of deadlines as compared to Greedy approach.

[13] Proposes a workflow scheduling algorithm based on critical path heuristics which try to schedule the critical tasks first. Authors proposed SAAS partial critical path algorithm which tries to minimize the cost of execution of workflows within user specified deadlines. For performance evaluation, authors have used library of realistic workflows like Montage, Cyber shake etc. and compared it with deadline MDP algorithm which is one of the most citied algorithm in this area. Simulation results show that SAAS-PCP (partial critical path) algorithm performs better than the deadline MDP algorithm which reduces the cost of execution of workflows within user specified deadlines.

[14] Proposes workflow scheduling algorithm based on budget and deadline trying to optimize both cost and time. It reduces execution cost while meeting user defined deadlines for delivering results and checking the behaviour of the algorithm. Authors divided the tasks into synchronization task or simple task and performed experiments on randomly generated workflows and tried to optimize cost and time for these workflows. Deadline and budget are distributed in this algorithm based on the minimum execution time and minimum cost. The objective of the proposed scheduling algorithm is to develop workflow schedule which meets user defined deadlines along with execution cost.

[15] proposes new algorithm for ensembles on Infrastructure- as-a Service (IaaS) regarding their management under deadline and budget constraint. Authors design Dynamic Provisioning Dynamic Scheduling (DPDS) algorithm which is online algorithm, providing schedules and 
resources at run time. In this ensembles are created using the synthetic workflows from the real applications like LIGO, CyberShake etc. Overall, three different algorithms are designed: DPDS and Workflow-Aware DPDS (WA-DPDS) which are dynamic algorithms, and one static algorithm: Static Provisioning Static Scheduling (SPSS). The simulation results show that SPSS and WA-DPDS (which takes into account the structure of workflows and task runtime) give better result than the simple priority based scheduling which makes decisions on the basis of resource utilization only. These algorithms are analyzed on CloudSim simulator which models the infrastructure and the application, taking into account the uncertainties in task runtime. The objective of this work is to maximize the number of user-prioritized workflows that can be completed within given budget and deadline constraints.

\section{Motivation}

Workflow scheduling is the major problem area in workflow management. There are many algorithms proposed by different researchers for workflow scheduling in Clouds. Some metaheuristics like Genetic Algorithm (GA), Particle Swarm Optimization (PSO) and Ant-Colony Optimization (ACO) already exist for workflow scheduling. Every algorithm has its own strategy while scheduling workflow application. Algorithms assign resources to workflow application based on the priority of hardware resources or tasks. We felt that capability of hardware resources and performance requirement should be considered while assigning resources to workflow application. We are motivated by the score concept [16] to measure hardware capability and to calculate minimum performance requirement for workflow tasks execution. Microsoft is using the WINSAT (Window System Assessment Tool) module in Windows to measure the hardware capabilities it is running in terms of WEI (Windows Experience Index) score [16]. We felt, the score concept can be used in Cloud environment also to measure the capabilities of hardware resources as score represents the minimum performance of the system and minimum threshold performance requirement of the workflow tasks. Score based scheduling will allow us to schedule workflow applications on high performance reliable machines which will lead to reduced failure of workflow applications. It also helps to meet user imposed deadline constraint with manageable cost. We found no evidence of this kind of work performed earlier. The score based workflow scheduling algorithm finds a schedule which meets the user defined deadline constraint and provide reliable machines for workflow execution within budget.

\section{ALGORITHM DESCRIPTION}

Our main purpose is to schedule the workflow application over reliable machines such that execution time can be minimized with manageable cost within user specified deadline constraint. The Score based workflow scheduling algorithm selects only those machines for scheduling which satisfy minimum threshold of workflow tasks. Figure 2 describes the flow chart of Score based deadline constraint workflow scheduling algorithm. 
International Journal on Cloud Computing: Services and Architecture (IJCCSA) ,Vol.3, No.6, December 2013

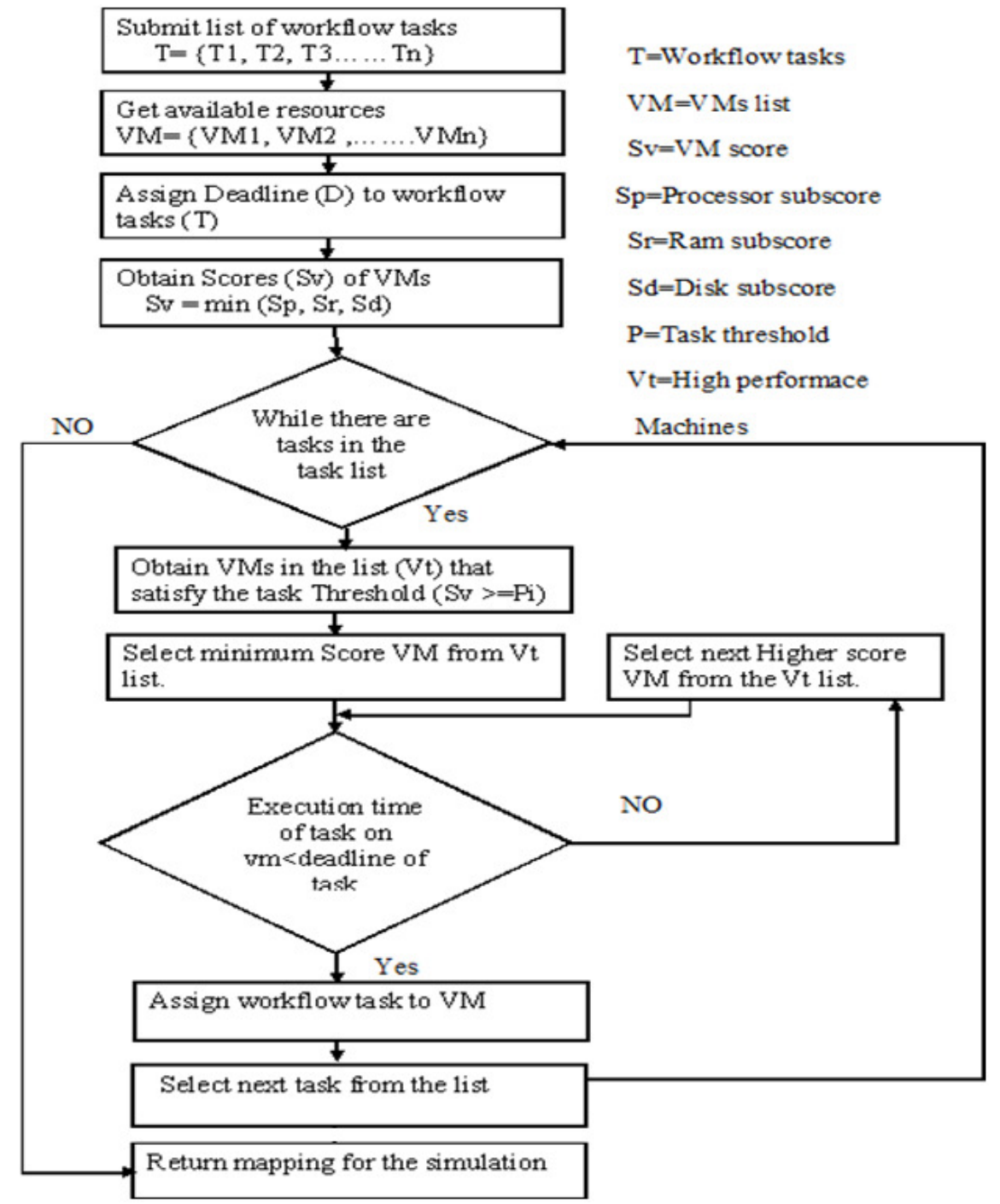

Figure 2. Flow chart of score based scheduling algorithm

The steps of the algorithm are described below:

Step 1: Submit the list of workflow tasks. i.e. T=T1,T2,T3 ...Tn.

Step 2: Get the available virtual resources from data center. i.e. VM=VM1, VM2, VM3 ..........VMn.

Step 3: Impose user defined deadline constraint $D$ on the entire workflow application in the form of sub deadlines of tasks.

Step 4: Obtain the final scores of the VMs from the components minimum sub-scores.

Step 5: While task list has the tasks to schedule, repeat steps 6,7 and 8 otherwise return the mapping of resources to tasks.

Step 6: Pick the minimum score VM from the VM list that satisfies the threshold of the task. Task threshold is defined on the basis of its instruction length.

Step 7: If the selected VM can execute the task within specified deadline then task is assigned to the selected VM otherwise next minimum score VM is selected from the list of resources.

Step 8: Select the next task from the list. If all tasks are scheduled then their mapping to VMs is returned for the simulation. 


\subsection{Score}

For the manageable cost, the proposed algorithm chooses the low score resources that will satisfy the minimum task threshold and execute the task within the deadline constraint. For obtaining the score of the machines it uses the concept of Microsoft WEI [16]. Score is used to define the capability of the machine. Each hardware component receives an individual score and final score of the machine is determined by lowest sub score of the component. The final score represents the minimum performance of the machine, based on the capabilities of the different parts of the machine including processing power, storage space and RAM. As shown in Table 1 final score of the machine is determined by the lowest sub score of the component. Individual sub-score of the components are defined on the basis of their calculations per second, memory operation per second and data transfer rate. More are the calculations, memory operations and data transfer rate, more is the score of individual component. Final score of the machine is obtained by the lowest sub score of the component. Similarly, minimum threshold of the tasks is defined on the basis of task score. Task threshold is obtained by number of instructions in the task. Higher complexity of the task leads to higher task score (threshold). So proposed algorithms looks for machines having score equal to or more than the threshold and execute the workflow application under the specified deadline constraint.

Table 1. Calculating score of machine

\begin{tabular}{|l|l|l|l|}
\hline Component & What is rated & Sub score & Final score \\
\hline Processor & $\begin{array}{l}\text { Calculation per second } \\
\text { (Mips) }\end{array}$ & 6 & 4 \\
\cline { 1 - 2 } Ram (memory) & $\begin{array}{l}\text { Memory operation per } \\
\text { second }\end{array}$ & 5 & $\begin{array}{l}\text { (Determined by lowest } \\
\text { sub score) }\end{array}$ \\
\cline { 1 - 3 } Storage space & Disk data transfer rate & 4 & \\
\hline
\end{tabular}

\subsection{Deadline}

User specified deadline D is achieved via distributing the entire deadline D over the workflow application as shown in Figure 3. On the basis of deadline D, algorithm calculates the sub deadlines $\mathrm{d} 1, \mathrm{~d} 2$, and $\mathrm{d} 3$ of the tasks and tries to schedule the task within that sub deadline of the task. Thus entire workflow application is executed within user specified deadline D e.g. let the task T1, T2 and T3 of the workflow application having 1000, 5000, and 4000 instructions and user specified deadline of $100 \mathrm{Hrs}$. Then the sub deadlines of the tasks T1, T2 and T3 are calculated on the basis user specified deadline and number of instructions of the tasks. Thus for T1 sub deadline is $10 \mathrm{Hrs}$, for T2 sub deadline is $40 \mathrm{Hrs}$ and for T3 the sub deadline is $50 \mathrm{Hrs}$. The algorithm tries to schedule the tasks within their sub deadlines so that entire workflow application is executed within $100 \mathrm{Hrs}$.

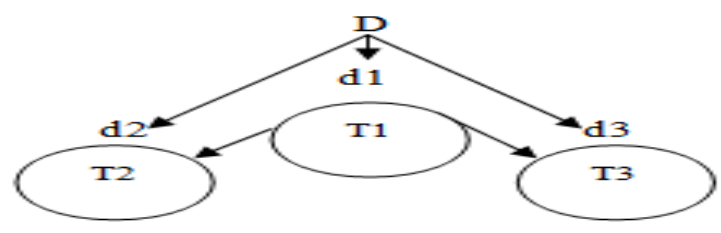

Figure 3. Deadline distribution 


\section{EXPERIMENTAL RESULTS}

For performance analysis, two algorithms have been designed. One is the same as described in the previous section and makes use of score whereas another one is without score. Both these algorithms have been simulated using CloudSim [17] on Windows 7 OS using Eclipse with Java version 1.6. Both the algorithms have been executed using sample workflow applications with different number of workflow application tasks. The desired scheduling is the one which executes the workflow within user specified deadline and with minimum execution cost. Here execution cost refers to the cost of virtual machines for executing the workflow tasks over them. Each VM has been assigned a cost based on the configuration of the machine. Better configuration machines exhibit more cost as compared to lower configuration machines. While performing experiments, we noted the execution cost, execution time and failure rate of workflows with both the algorithms. Both the algorithms have been run by varying the number of cloudlets (tasks) in workflow as well as by varying the number of iterations. Experimental results show that score based algorithm exhibits less execution time and reduces failure rate with manageable cost as compared to other algorithm which is not using the concept of scores.

Figure 4 shows the execution cost of workflow by varying the number of cloudlets for both the algorithms with fixed deadline. Results show that in score based deadline constraint algorithm, execution cost does not increase very much compared to without score algorithm and takes just little more cost as compared to without score deadline constraint algorithm.

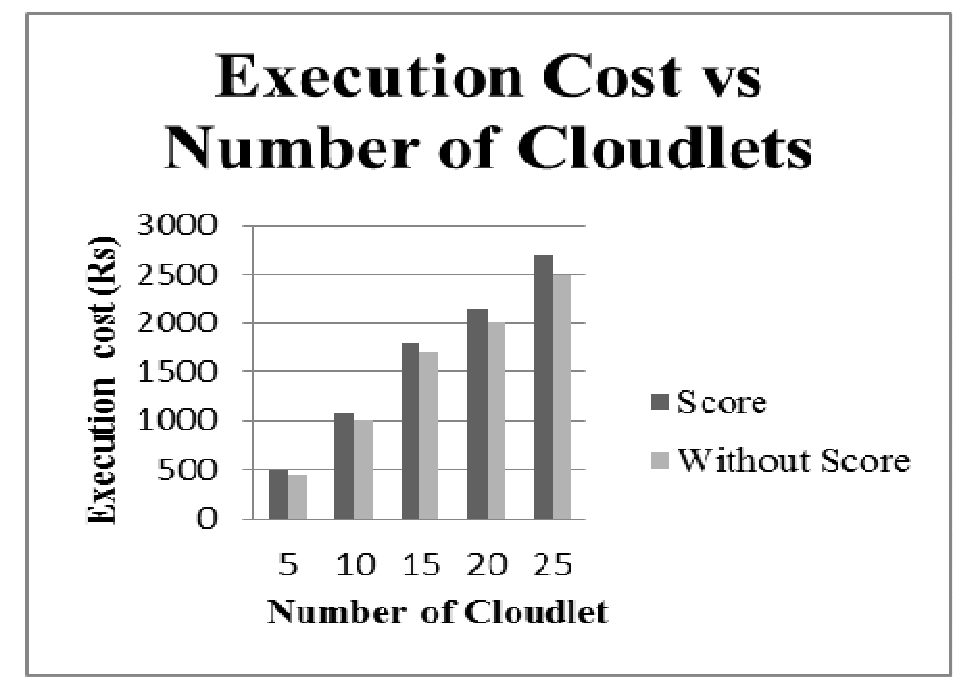

Figure 4. Execution cost vs. number of cloudlets

Figure 5 shows the execution time of workflow by varying the number of cloudlets. Results show that score based deadline constraint algorithm reduces the execution time as compared to without score deadline constraint algorithm. 


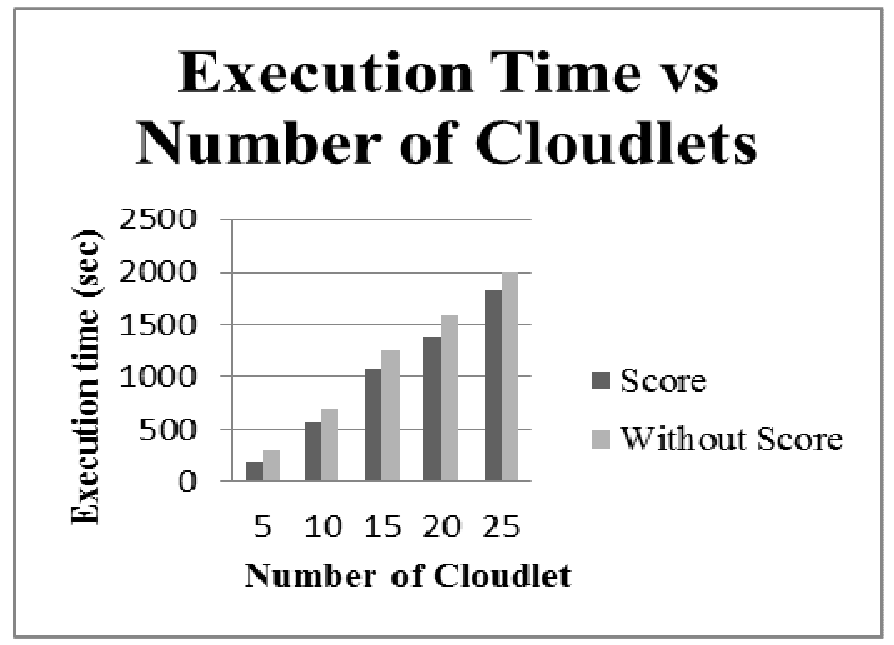

Figure 5. Execution time vs. number of cloudlets

Score based deadline constraint scheduling algorithm also reduces the failure rate of workflow applications as this algorithm schedules the workflow tasks only on the high performance reliable machines. Failure rate of workflow is calculated on the basis of ratio of total number of times virtual machines fails to execute the workflow application with the total number of times workflow application is executed. Proposed algorithm reduces the failure rate of workflow applications by considering only those virtual machines for scheduling for which minimum performance is more than the required performance (task threshold) of the workflow tasks and filter out the low performance machines that do not satisfy the threshold criteria of the workflow tasks.

Figure 6 shows the failure rate of workflows. Results show that score based deadline constraint workflow scheduling algorithm reduces the failure rate as compared to without score based deadline constraint workflow scheduling algorithm.

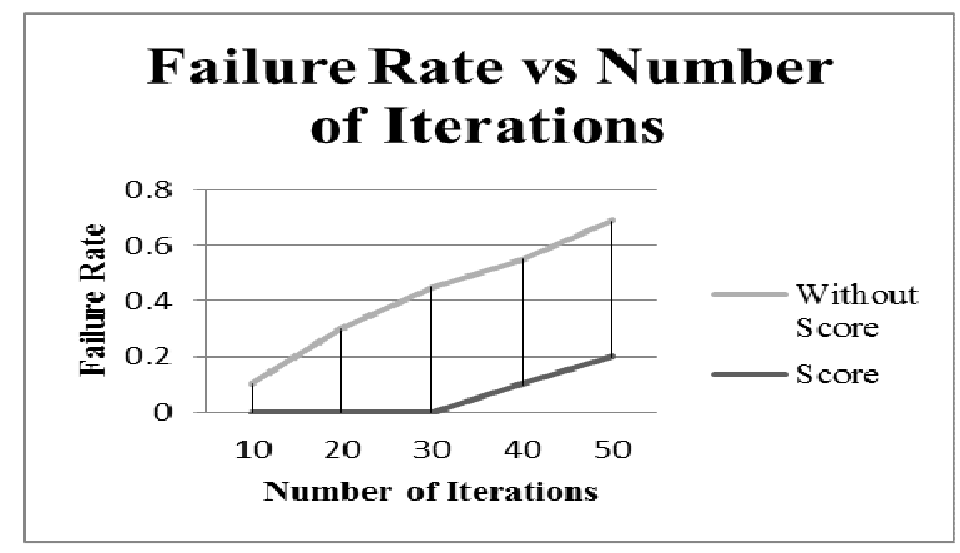

Figure 6. Failure rate vs. number of iterations 


\section{CONCLUSION AND FUTURE SCOPE}

The Cloud computing model enable users to use services as utilities. It allows users to access these services over the Internet and make payments according to the usage and level of quality requested by the user. In this research work, we have proposed a score based deadline constraint workflow scheduling algorithm that achieves less execution time along with manageable execution cost under the user specified deadline constraint. We also compared it with withoutscore deadline constraint scheduling algorithm. Experimental results show that proposed algorithm also reduces the failure rate of workflow applications.

In future we are planning to introduce the concept of score in existing heuristics techniques which may improve the performance of these techniques. We are also trying to extend our work to support real time workflows.

\section{REFERENCES}

[1] R.Buyya, C.S Yeo, S.Venugopal, J. Broberg, and I. Brandic, (2009) "Cloud Computing and Emerging IT platforms: Vision, Hype, and Reality for Delivering Computing as the 5th Utility", Journal of Future Generation Computer Systems, Vol.25, No. 6, pp. 599-616.

[2] J. Geelan, "Twenty one experts define Cloud computing Virtualization", Electronic magazine, [Online] Available at http:// virtualization.sys.con.com/node/612375.

[3] L. Mei, W.K. Chan, T.H. Tse, (2008) "A Tale of Clouds: Paradigm Comparisons and some Thoughts on Research Issues”, IEEE Asia-Pacific Services Computing Conference (APSCC), pp. 464-469.

[4] J. Yu, R. Buyya, (2008) "Workflow scheduling Algorithms for Grid computing", metaheuristics for scheduling in distributing computing environment, Springer, Berlin.

[5] Y. Zhang, A. Mandal, C Koelbel, K. Cooper, (2009) "Combined Fault Tolerance and Scheduling Techniques for Workflow Application on Computational Grids", 9th IEEE/ACM international Symposium on Clustering and Grid.

[6] J.Yu R. Buyya, (2006) "Scheduling Scientific Workflow Applications with Deadline and Budget Constraints using Genetic Algorithms", Scientific Programming Journal, pp. 217-230.

[7] M. Xu, L. Cui, H. Wang, Y. Bi, (2009) "A Multiple QoS Constrained Scheduling Strategy of Multiple Workflows for Cloud Computing”, IEEE International Symposium on Parallel and Distributed Processing with Applications.

[8] J. zhang W. neng chen., (2009) "An Ant Colony Optimization Approach to a Grid Workflow Scheduling Problem With Various QoS Requirements", IEEE Transactions on Systems, Man, and Cybernetics.

[9] J. Nirmala, S. Bhanu, S. Jayadivya, (2010) "Fault tolerant workflow scheduling based on replication and resubmission of tasks in Cloud Computing", International Journal on Computer Science and Engineering.

[10] L. Wu, S. Panday, R. Buyya, (2010) "A Particle Swarm Optimization-Based Heuristic for Scheduling Workflow Applications in Cloud Computing Environments", 24th IEEE International Conference on Advanced Information Networking and Applications.

[11] K.Liu, H. Jin, J. Chen, X. Liu, D.Yuan, Y. Yang, (2010) “A Compromised Time-Cost Scheduling Algorithm in SwinDeW-C for Instance-Intensive Cost-Constrained Workflows on Cloud Computing Platform", International Journal of High Performance Computing Applications , pp. 445-456.

[12] L. Fernando, B. Edmundo, M. Madeira M, (2011) "HCOC: A Cost Optimization Algorithm for Workflow Scheduling in Hybrid Clouds", Journal of Internet Services and Applications, Springer, pp. 207-227.

[13] M. Naghibzadeh, S. Abrishami, (2011) "Deadline-constrained workflow scheduling in software as a service Cloud”, Scientia lranica, pp. 680-689.

[14] S. Kaushal, A. Verma, (2012) "Deadline and Budget Distribution based Cost- Time Optimization Workflow Scheduling Algorithm Cloud”, International Journal of Computer Applications.

[15] M. Malawski, G. Juve, E. Deelman and J. Nabrzyski, (2012) "Cost and Deadline Constrained Provisioning for Scientific Workflow Ensembles in IaaS Clouds”, IEEE International Conference. 
International Journal on Cloud Computing: Services and Architecture (IJCCSA), Vol.3, No.6, December 2013

[16] "Windows System Tools," [Online]. Available at http: //www.en.wikipedia.org/wiki/ Windows_System_Assessment_Tool.

[17] R. Ranjan, A. Beloglazov, A. F. De Rose, R. Buyya, N. Calheiros, (2011) "CloudSim: A Toolkit for Modeling and Simulation of Cloud Computing", Software: Practice and Experience (SPE), Wiley Press, Vol. 41, No. 1, pp. 23-50.

\section{Authors}

Ranjit Singh received his B.Tech degree in Computer Science and Engineering from Punjab Technical University, Jalandhar, Punjab, India. Currently he is pursuing M.E degree in Computer Science and Engineering from Panjab University, Chandigarh, India. His research interests include Cloud Computing and scheduling issues in distributed environments.

Sarbjeet Singh received his B.Tech degree in Computer Science and Engineering from Punjab Technical University, Jalandhar, Punjab, India in 2001 and M.E. degree is Software Engineering from Thapar University, Patiala, India in 2003. He also received Ph.D degree in Computer Science and Engineering from Thapar University, Patiala, India in 2009, working on grid security systems architecture.
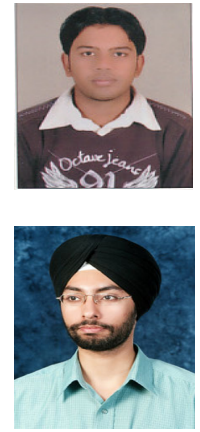

Currently he is working as Associate Professor in Computer Science and Engineering at UIET, Panjab University, Chandigarh, India. He has more than 35 research publications in various conferences and journals to his credit. His research interests include parallel and distributed systems, distributed security architectures, distributed services like grid and web services, privacy and trust related issues in distributed environments. Dr. Singh is a life member of Computer Society of India and Indian Society for Technical Education. 